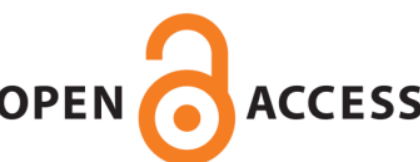

OPEN ACCESS

UWS Academic Portal

\title{
The effectiveness of a Sierpinski carpet-inspired transducer
}

Koubayssi, Sara; Walker, Alan; Roach, Paul

Published in:

Fractals

DOI:

$10.1142 / S 0218348 \times 17500505$

Published: 01/09/2017

Document Version

Peer reviewed version

Link to publication on the UWS Academic Portal

Citation for published version (APA):

Koubayssi, S., Walker, A., \& Roach, P. (2017). The effectiveness of a Sierpinski carpet-inspired transducer. Fractals, 25(5), [1750050]. https://doi.org/10.1142/S0218348X17500505

\section{General rights}

Copyright and moral rights for the publications made accessible in the UWS Academic Portal are retained by the authors and/or other copyright owners and it is a condition of accessing publications that users recognise and abide by the legal requirements associated with these rights.

\section{Take down policy}

If you believe that this document breaches copyright please contact pure@uws.ac.uk providing details, and we will remove access to the work immediately and investigate your claim. 


\title{
THE EFFECTIVENESS OF A SIERPINSKI CARPET INSPIRED TRANSDUCER
}

\author{
Running Title: Fractal Ultrasonic Transducers \\ S. Canning ${ }^{\dagger}$, A. J. Walker ${ }^{\S *}$ and P. A. Roach ${ }^{\dagger}$ \\ $\dagger$ School of \\ $\S$ School of \\ Computing and Mathematics, \\ Science and Sport, \\ University of South Wales, University of the West of Scotland, \\ Pontypridd, \\ Paisley, \\ CF37 1DL, \\ PA1 2BE, \\ United Kingdom \\ United Kingdom
}

June 8, 2017

keywords: Ultrasound; Sierpinski Carpet; Sierpinski Gasket; Ultrasonic Transducer; Green Function Renormalization

e-mail: sara.koubayssi@southwales.ac.uk, alan.walker@uws.ac.uk, paul.roach@southwales.ac.uk

\begin{abstract}
Piezoelectric ultrasonic transducers have the ability to act both as a receiver and a transmitter of ultrasound. Standard designs have a regular structure and therefore operate effectively over narrow bandwidths due to their single length scale. Naturally occurring transducers benefit from a wide range of length scales giving rise to increased bandwidths. It is therefore of interest to investigate structures which incorporate a range of length scales, such as fractals. This paper applies an adaptation of the Green function renormalization method to analyse the propagation of an ultrasonic wave in a series of pre-fractal structures. The structure being investigated here is the Sierpinski carpet. Novel expressions for the non-dimensionalized electrical impedance and the transmission and reception sensitivities as a function of the operating frequency are presented. Comparisons of metrics between three new designs alongside the standard design (Euclidean structure) and the previously investigated Sierpinski gasket device are performed. The results indicate a significant
\end{abstract}

\footnotetext{
${ }^{*}$ Corresponding author.
} 
improvement in the reception sensitivity of the device, and improved bandwidth in both the receiving and transmitting responses.

\section{INTRODUCTION}

Transducers are devices which enable the conversion of electrical energy into mechanical energy and vice-versa. Ultrasonic transducers are used for the generation and detection of ultrasonic waves. The uses of such devices vary extensively and they are routinely used in applications including communication, medical diagnosis and non-destructive testing $[1,2]$. There are two main types of ultrasonic transducers that are used in generating and detecting ultrasonic waves; these are electrostatic and piezoelectric transducers. Electrostatic devices employ an oscillating membrane connected to a backplate which can incorporate resonating conduits and/or cavities [3-7]. Electrostatic devices generally have higher sensitivities and bandwidth however, they suffer from requiring a higher operating voltage then piezoelectric devices [8]. This paper focuses on the modelling of novel piezoelectric ultrasonic transducers.

Piezoelectric transducers have a piezoelectric material sandwiched between a backing layer and front matching layer. The conversion of energy in these transducers is made possible by the piezoelectric ceramic. During reception mode, the ceramic expands and contracts through the application of a mechanical stress causing the creation of an electrical current; Fig. 1(a). In transmission mode, the continuous expansion and contraction of the material when an electric current is applied results in the production of mechanical vibrations; see Fig. 1 (b).

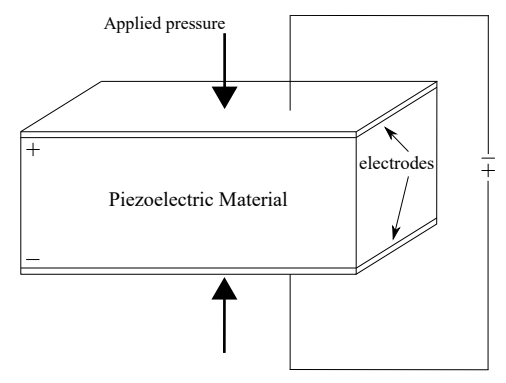

(a) The piezoelectric element produces a voltage when subject to pressure at its plates.

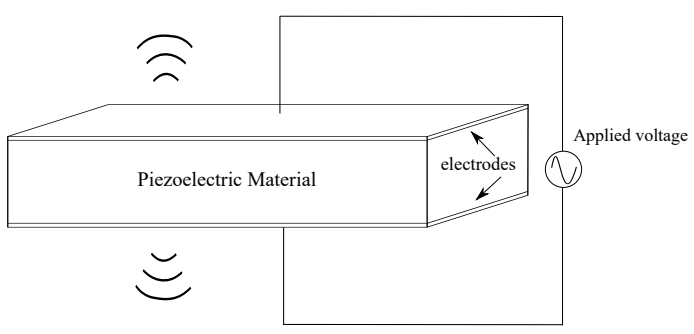

(b) The piezoelectric element generates vibrations when applied with a voltage.

Figure 1: Schematic of transducer in (a) reception mode, and (b) transmission mode 
The geometry of traditional ultrasonic transducers is regular and is generally manufactured from a piezoelectric crystal which is diced and filled with a polymer; see Fig. 2. Such designs have one dominant length scale and consequently only perform efficiently over a small range of frequencies. In contrast the transducers found in natural systems have complexity in their shapes, with resonators over a range of length scales [9-11]. As a result, they are far more efficient in transmitting and receiving ultrasonic waves over a range of frequencies than man-made devices. Thus, it is of great interest to construct devices which are inspired by those found in nature. This may be achieved through the development of more intricate designs.

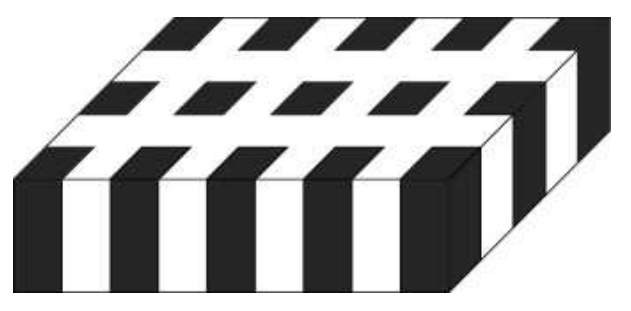

Figure 2: 1-3 Ceramic-polymer composite transducer design.

Fractals can be characterized as complex objects which exhibit similarity at any magnification in addition to covering a range of length scales. The implementation of fractal-like structures into the design of new ultrasonic transducers can assist the construction of devices that can operate efficiently over a large range of frequencies. This paper is therefore concerned with the design and mathematical modelling of a range of novel piezoelectric ultrasonic transducers that incorporate pre-fractal structures.

Research on fractal inspired transducers has commenced [9, 12-14]. In particular the plane wave expansion (PWE) model was employed to study the performances of a Sierpinski carpet type and Cantor set transducers [12]. Furthermore, analytical results for a Sierpinski gasket-inspired transducer were obtained in [9] using the Green function renormalization method. The Sierpinski gasket transducer was further investigated using a finite element approach [14] and a prototype for a specified fractal generation was manufactured [13]. In these papers the results agreed with the initial assumption that the introduction of multiple length scales into the design of ultrasonic transducers improves effective operation over a larger range of frequencies with resonating behaviour.

In this paper the Green function renormalization method is employed to analyse the 
propagation of an ultrasonic wave within an infinitely ramified structure. Three models based on the design of the Sierpinski carpet pre-fractal are investigated to determine the most appropriate device for ultrasonic applications. We find a significant improvement for the reception sensitivity of the device, and improved bandwidth in both the receiving and transmitting responses.

Section 2 outlines the construction of the Sierpinski carpet pre-fractal along with the derivation of its lattice counterpart. In Sec.3, the Green function renormalization method is utilized to obtain the relevant relations for each transducer model. Boundary conditions are obtained to aid the derivation of the expressions for the important output parameters, electrical impedance and transmission and reception sensitivities in Sec. 4 . In Sec. 5, the results for the individual Sierpinski carpet transducer models are compared with the standard Euclidean transducer and the Sierpinski triangle transducer. The findings are summarized and future research is discussed in Sec. 6 .

\section{FORMULATION OF THE MODEL}

The fractal used here to imitate the complex geometry found in natural ultrasonic transducers is the Sierpinski carpet. The structure of this fractal begins with a square, which is then copied and scaled into nine congruent sub-squares with the centre square eliminated. The following generation is then achieved by replacing the initial square with this newly formed shape. Subsequent generations are then found by repeatedly applying this procedure to give the Sierpinski carpet [15-17], see Fig. 3. In actuality the structures formed from the iterative process are pre-fractals as the fractal is only formed after an infinite number of iterations [18]. For the purpose of manufacturing such designs, it is only the pre-fractals that are of interest.
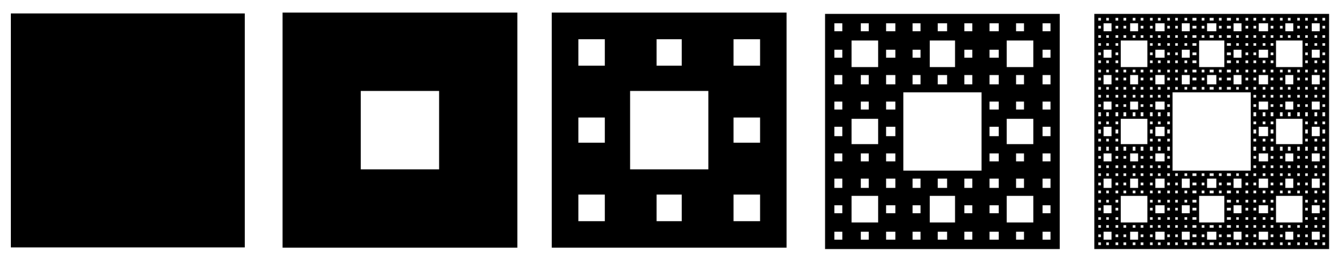

Figure 3: The initial square and first four iterations of the Sierpinski carpet. 
A lattice equivalent of the Sierpinski carpet can be obtained by considering the fractal structures as a sequence of graphs $\left\{G^{(n)}\right\}$, where the superscript $n$ refers to a particular fractal generation level[9, 19, 20]. The subsequent generation level graph, $G^{(n+1)}$, is then obtained by connecting together eight copies of $G^{(n)}$. The $n$th generation graph has $N_{n}=2^{3 n}$ vertices, and $L$ is the side length of the structure which is assumed to be fixed throughout the construction process [9]. As a result of this condition, the edge length between adjacent vertices will reduce to zero, as the generation level is increased $[19,21]$. For the sequence of graphs the coordination number $q$ of the lattice refers to the vertex degree of the graph. As such $q$ is dependent on the generation level as it is determined by the number of sub-graphs it connects. Consequently, the number of connection vertices will increase as the generation level is increased (Fig. 4). Thus, the Sierpinski carpet lattice has coordination number 4 for vertices connecting three subgraphs, 3 for vertices connecting two sub-graphs and 2 for non-connecting vertices. As a result, the input/ output vertices have coordination number 2 , which remains constant throughout the iteration process. Similar to the work presented in [9], fictitious vertices $A, B, C$ and $D$ are attached to these vertices to accommodate the boundary conditions, see Fig. 5.

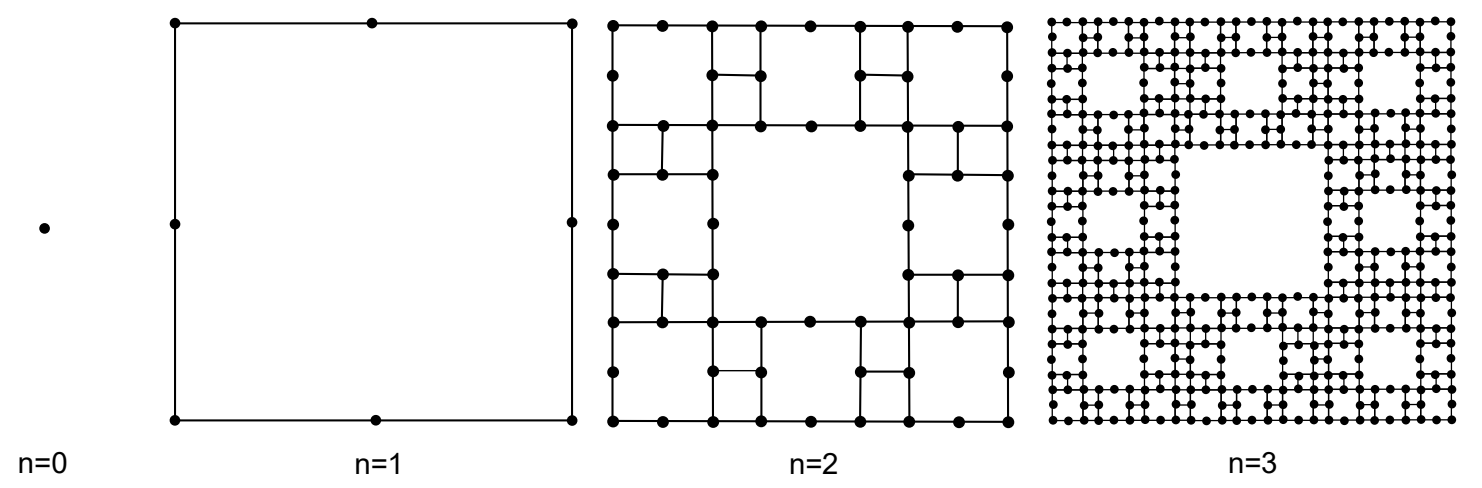

Figure 4: Graphical representations of generations 0 to 3 for the sequence of Sierpinski carpet lattices.

Each graph of the sequence can be completely described by its adjacency matrix $H^{(n)}$, where the $(i, j)^{\text {th }}$ element gives the number of edges connecting vertex $i$ to $j$ by assigning the value one to connected vertices and zero otherwise. The recursive 
relationship of the graphs is then given as $[9,22]$

$$
H^{(n+1)}=\bar{H}^{(n)}+V^{(n)},
$$

where $\bar{H}^{(n)}$ is a block diagonal matrix which represents the connectivity properties of the individual sub-graphs of $G^{(n)}$. The connection of these sub-graphs is given by $V^{(n)}$ which is a sparse matrix that assigns the number one to the connection of vertices on sub-graphs.

The one-dimensional piezoelectric constitutive equations are required to describe the interaction between the elastic variables, stress $T$ and strain $S$, to electric variables, field $E$ and displacement $D$, within the piezoelectric material. These are of the form,

$$
\begin{aligned}
& T=Y S-h D, \\
& E=-h S+\frac{D}{\epsilon},
\end{aligned}
$$

where $Y$ represents the Young's modulus, $h$ is the piezoelectric constant and $\epsilon$ is the permittivity. The analysis of a propagating ultrasonic wave within the Sierpinski carpet lattice requires the use of the discretized wave equation [9],

$$
\rho_{T} \frac{\partial^{2} \underline{u}}{\partial t^{2}}=\frac{Y_{T}}{\Delta x^{2}}\left(A^{(n)} \underline{u}+B^{(n)} \underline{u}+\underline{c}^{(n)}\right)
$$

where $\rho_{T}$ and $Y_{T}$ are the density and Young's modulus for the piezoelectric material respectively, $u$ is the particle displacement, $\Delta x$ is the distance between neighbouring vertices, $A^{(n)}$ is the matrix representing the discretized Laplacian and $B^{(n)}$ and $\underline{c}^{(n)}$ are a matrix and vector containing the boundary conditions at the input and output vertices. Previously $[9,19,22-24]$, it was established that a method for solving the discretized wave equation, Eq. (2.4), is achieved by initially neglecting boundary conditions. These boundary conditions are then re-introduced later in the analysis, by means of suitable matrix transformations. Non-dimensionalizing and transforming Eq. (2.4) 
into the Laplace domain gives

$$
\begin{gathered}
\left(p^{2} I_{n}-A^{(n)}-B^{(n)}\right) \underline{u}=\underline{c}, \\
\Rightarrow \underline{u}=G^{(n)} \underline{c},
\end{gathered}
$$

where $p$ is the Laplace transform variable, $I_{n}$ is an $n \times n$ identity matrix and $G^{(n)}$ is the Green function matrix given by

$$
G^{(n)}=\left(p^{2} I_{n}-A^{(n)}-B^{(n)}\right)^{-1} .
$$

The Green function matrix not accounting for boundary conditions is thus given by

$$
\hat{G}^{(n)}=\left(p^{2} I_{n}-A^{(n)}\right)^{-1}
$$

Furthermore, the Laplacian matrix $A^{(n)}$, is given by $A^{(n)}=H^{(n)}-q I_{n}$ and so utilising Eqs.(2.1) and (2.7) results with the following recursion relationship equation [20, 22, 23],

$$
\hat{G}^{(n+1)}=\bar{G}^{(n)}+\bar{G}^{(n)} V^{(n)} \hat{G}^{(n+1)},
$$

where $\bar{G}^{(n)}$ is a block diagonal matrix whose $v$ blocks equal $\hat{G}^{(n)}$. Similarly, utilising Eqs. (2.1) and (2.6), the following relationship is obtained [20,23]

$$
G^{(n)}=\hat{G}^{(n)}+\hat{G}^{(n)} B^{(n)} G^{(n)} .
$$

Due to the symmetries of the lattice structure, it is of interest to only obtain the pivotal Green functions. The pivotal Green functions are the minimum number of independent elements required to develop a recursion [20]. The next section will develop these pivotal Green functions for each transducer model using Eq. (2.9). 


\section{RENORMALIZATION ANALYSIS}

This section develops the Green function renormalization to infinitely ramified Sierpinski carpets. It is clear that the number of output vertices the transducer exhibits will have a direct effect on its performance, and as such it is desirable to obtain individual models which encompass these different numbers of output vertices. In transmission mode, each model will contain a single input vertex that is connected to fictitious vertex $A$. At this vertex ultrasonic waves are generated through the application of an electric field and dependent on the model, one, two or three output vertices will be required. These output vertices will produce the mechanical vibrations once the wave has passed through the transducer.

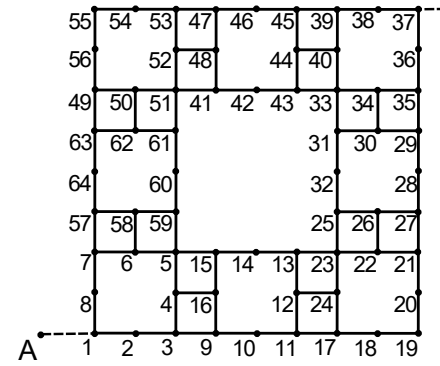

(a) Model $\eta$

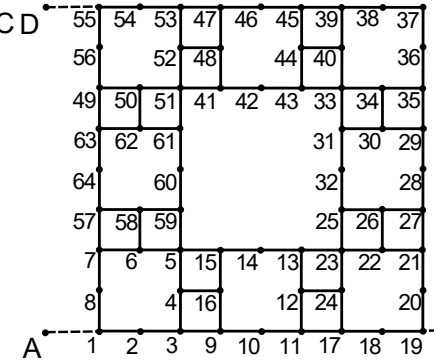

(b) Model $\delta$

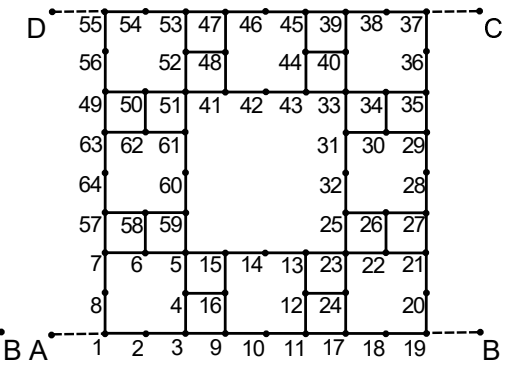

(c) Model $\gamma$

Figure 5: Sierpinski carpet lattice at generation $n=2$. Fictitious vertices $A, B, C$ and $D$ are introduced to accommodate the boundary conditions. Representation of (a) Model $\eta$ with fictitious vertices $A$ and $C$, (b) Model $\delta$ with fictitious vertices $A, B$ and $D$, and (c) Model $\gamma$ with fictitious vertices $A, B, C$ and $D$.

\subsection{Model $\eta$ : Single Output Vertex}

The graph in Fig. 5 (a) displays model $\eta$ at generation level two. As illustrated, model $\eta$ contains two boundary conditions; an input vertex placed at vertex 1 and a single output vertex placed at vertex 37 . As a result fictitious vertices $A$ and $C$ will be attached to these vertices. Thus, model $\eta$ requires the following pivotal elements: $G_{11}^{(n)}, G_{137}^{(n)}$ and $G_{3737}^{(n)}$. These can more conveniently be expressed as $x_{\eta}, z_{\eta}$ and $v_{\eta}$, respectively, where the subscript relates to the transducer model rather than to the vertices of the lattice. By utilizing Eq. (2.9), the following system of equations for the pivotal Green functions 
were obtained

$$
\begin{aligned}
& x_{\eta}=\hat{x}_{\eta}+\hat{x}_{\eta} b_{1, \eta} x_{\eta}+\hat{z}_{\eta} b_{2, \eta} z_{\eta}, \\
& z_{\eta}=\hat{z}_{\eta}+\hat{z}_{\eta} b_{1, \eta} x_{\eta}+\hat{x}_{\eta} b_{2, \eta} z_{\eta}, \\
& v_{\eta}=\hat{x}_{\eta}+\hat{z}_{\eta} b_{1, \eta} z_{\eta}+\hat{x}_{\eta} b_{2, \eta} v_{\eta},
\end{aligned}
$$

where $\hat{x}_{\eta}=\hat{G}_{11}^{(n)}$ and $\hat{z}_{\eta}=\hat{G}_{137}^{(n)}$ correspond to the boundary matrix, $B^{(n)}$, being set to zero, and $b_{1, \eta}=B_{11}^{(n)}$ and $b_{2, \eta}=B_{3737}^{(n)}$ relate to the boundary conditions set at fictitious vertices $A$ and $C$. Thus solving these three equations solely in terms of $\hat{x}_{\eta}, \hat{z}_{\eta}, b_{1, \eta}$ and $b_{2, \eta}$ yields

$$
\begin{aligned}
& x_{\eta}=\frac{\left(\hat{z}_{\eta}^{2}-\hat{x}_{\eta}^{2}\right) b_{2, \eta}+\hat{x}_{\eta}}{\left(\left(\hat{x}_{\eta}^{2}-\hat{z}_{\eta}^{2}\right) b_{1, \eta}-\hat{x}_{\eta}\right) b_{2, \eta}+1-\hat{x}_{\eta} b_{1, \eta}}, \\
& z_{\eta}=\frac{\hat{z}_{\eta}}{\left(\left(\hat{x}_{\eta}^{2}-\hat{z}_{\eta}^{2}\right) b_{1, \eta}-\hat{x}_{\eta}\right) b_{2, \eta}+1-\hat{x}_{\eta} b_{1, \eta}}, \\
& v_{\eta}=\frac{\left(\hat{z}_{\eta}^{2}-\hat{x}_{\eta}^{2}\right) b_{1, \eta}+\hat{x}_{\eta}}{\left(\left(\hat{x}_{\eta}^{2}-\hat{z}_{\eta}^{2}\right) b_{1, \eta}-\hat{x}_{\eta}\right) b_{2, \eta}+1-\hat{x}_{\eta} b_{1, \eta}} .
\end{aligned}
$$

In [19], one of the conditions in order to apply this renormalization method to a family of graphs is that the process of obtaining $G^{(n+1)}$ from $G^{(n)}$ consists of connecting together $v$ copies of $G^{(n)}$ solely through the input/ output vertices. For the Sierpinski gasket inspired device Eq.(2.8) was suffice in obtaining exact matrix elements for subsequent generation levels. This was suitable since this structure is a finitely ramified fractal and so the connection process only involved the input/ output vertices. As the Sierpinski carpet is an infinitely ramified fractal, the connection process to form the $n^{\text {th }}$ generation level graph consists of connecting $v$ copies of $G^{(n)}$ at the input/ output vertices in addition to internal vertices. An alternative approach is therefore required to apply a similar methodology to a Sierpinski carpet inspired device. Instead the derivation of the Green function matrices are required to obtain the essential matrix elements. Consequently, results for higher generation levels are computationally intensive and therefore this paper will concentrate on low generation levels.

Appendix A concisely details the process of obtaining the required Green function elements for the first generation level of model $\gamma$ of the Sierpinski carpet pre-fractal. 


\subsection{Model $\delta$ : Two Symmetric Output Vertices}

The second generation graph of model $\delta$ is shown in Fig. 5 (b). This model will include three boundary conditions, an input vertex at vertex 1 and two symmetric output vertices positioned at vertices 19 and 55. Due to the symmetries of this lattice, it can be shown that $G_{119}^{(n)}$ and $G_{155}^{(n)}$ are equal. For this model it is necessary to determine the relations for $\hat{x}_{\delta}, \hat{y}_{\delta}, \hat{t}_{\delta}$ and $\hat{w}_{\delta}$, as it is assumed that vertex 37 is to be insulated from the external loads. The need for the addition of $\hat{t}$ and $\hat{w}$ is due to the symmetry of this lattice, see Fig. 5(b). In this instance $\hat{G}_{11}^{(n)} \neq \hat{G}_{1919}^{(n)}$. For brevity, the system of equations used to derive the pivotal Green functions for models $\delta$ and $\gamma$ is presented in Appendix B. Thus using analysis similar to that used for model $\eta$ (and employing Eqs. (B1)- (B4)) results with

$$
\begin{gathered}
x_{\delta}=\frac{\left(1-b_{2, \delta}\left(\hat{t}_{\delta}+\hat{w}_{\delta}\right)\right) \hat{x}_{\delta}+2 b_{2, \delta} \hat{y}_{\delta}^{2}}{\left(b_{2, \delta}\left(\hat{t}_{\delta}+\hat{w}_{\delta}\right)-1\right)\left(b_{1, \delta} \hat{x}_{\delta}-1\right)-2 b_{1, \delta} b_{2, \delta} \hat{y}_{\delta}^{2}}, \\
y_{\delta}=\frac{\hat{y}_{\delta}}{\left(b_{2, \delta}\left(\hat{t}_{\delta}+\hat{w}_{\delta}\right)-1\right)\left(b_{1, \delta} \hat{x}_{\delta}-1\right)-2 b_{1, \delta} b_{2, \delta} \hat{y}_{\delta}^{2}}, \\
w_{\delta}=\frac{b_{1, \delta} \hat{y}_{\delta}^{2}\left(1+2 b_{2, \delta}\left(\hat{t}_{\delta}-\hat{w}_{\delta}\right)\right)-\left(\hat{w}_{\delta}+b_{2, \delta} \hat{t}_{\delta}^{2}-b_{2, \delta} \hat{w}_{\delta}^{2}\right)\left(b_{1, \delta} \hat{x}_{\delta}-1\right)}{\left(1+b_{2, \delta}\left(\hat{t}_{\delta}-\hat{w}_{\delta}\right)\right)\left(\left(b_{2, \delta}\left(\hat{t}_{\delta}+\hat{w}_{\delta}\right)-1\right)\left(b_{1, \delta} \hat{x}_{\delta}-1\right)-2 b_{1, \delta} b_{2, \delta} \hat{y}_{\delta}^{2}\right)}, \\
t_{\delta}=\frac{\hat{t}_{\delta}-b_{1, \delta} \hat{t}_{\delta} \hat{x}_{\delta}+b_{1, \delta} \hat{y}_{\delta}^{2}}{\left(1+b_{2, \delta}\left(\hat{t}_{\delta}-\hat{w}_{\delta}\right)\right)\left(\left(b_{2, \delta}\left(\hat{t}_{\delta}+\hat{w}_{\delta}\right)-1\right)\left(b_{1, \delta} \hat{x}_{\delta}-1\right)-2 b_{1, \delta} b_{2, \delta} \hat{y}_{\delta}^{2}\right)},
\end{gathered}
$$

where $x_{\delta}, y_{\delta}, w_{\delta}$ and $t_{\delta}$ correspond to the pivotal elements $G_{11}^{(n)}, G_{119}^{(n)}, G_{1919}^{(n)}$ and $G_{1955}^{(n)}$ respectively.

\subsection{Model $\gamma$ : Three Output Vertices}

Model $\gamma$ refers to the inclusion of four boundary conditions, see Fig. 5 (c), where an input vertex is placed at vertex 1, two symmetric vertices are to be placed at vertices 19 and 55 and an additional output vertex is placed at vertex 37. For this model there are seven pivotal elements; $G_{11}^{(n)}, G_{119}^{(n)}, G_{137}^{(n)}, G_{1919}^{(n)}, G_{3737}^{(n)}, G_{1937}^{(n)}$ and $G_{1955}^{(n)}$, where for ease of notation these will be labelled respectively as $x_{\gamma}, y_{\gamma}, z_{\gamma}, w_{\gamma}, v_{\gamma}, u_{\gamma}$ and $t_{\gamma}$. Following 
with the same analysis as for models $\eta$ and $\delta$ results with the following seven equations,

$$
\begin{aligned}
& x_{\gamma}=\frac{\left(\begin{array}{r}
2 b_{2, \gamma}\left(\hat{x}_{\gamma}^{2}-\hat{y}_{\gamma}^{2}\right)+b_{2, \gamma}\left(\hat{x}_{\gamma}-\hat{z}_{\gamma}\right)\left(4 \hat{y}_{\gamma}^{2} b_{2, \gamma}-\hat{z}_{\gamma}\left(\hat{x}_{\gamma} b_{2, \gamma}-1\right)\right) \\
-b_{2, \gamma}^{2}\left(\hat{x}_{\gamma}^{3}-\hat{z}_{\gamma}^{3}\right)-x_{\gamma}
\end{array}\right)}{\Delta_{1}} \\
& y_{\gamma}=\frac{\hat{y}_{\gamma}\left(1-b_{2, \gamma}\left(\hat{x}_{\gamma}-\hat{z}_{\gamma}\right)\right)}{\Delta_{1}} \\
& z_{\gamma}=\frac{2 \hat{y}_{\gamma}^{2} b_{2, \gamma}+\hat{z}_{\gamma}\left(1-b_{2, \gamma}\left(\hat{x}_{\gamma}+\hat{z}_{\gamma}\right)\right)}{\Delta_{1}} \\
& w_{\gamma}=\frac{\left(\begin{array}{c}
\left(2 \hat{x}_{\gamma}^{2}\left(\hat{x}_{\gamma} b_{1, \gamma}-1\right)-2\left(2 \hat{y}_{\gamma}^{2}+\hat{z}_{\gamma}^{2}\right) b_{1, \gamma} \hat{x}_{\gamma}+\hat{y}_{\gamma}^{2}\left(1+4 \hat{z}_{\gamma} b_{1, \gamma}\right)+\hat{z}_{\gamma}^{2}\right) b_{2, \gamma} \\
-\left(\hat{x}_{\gamma}\left(\hat{x}_{\gamma}^{2} b_{1, \gamma}+\hat{x}_{\gamma}\left(\hat{z}_{\gamma} b_{1, \gamma}-1\right)-\left(4 \hat{y}_{\gamma}^{2}+\hat{z}_{\gamma}^{2}\right) b_{1, \gamma}-\hat{z}_{\gamma}\right)\right. \\
\left.+\hat{z}_{\gamma} b_{1, \gamma}\left(4 \hat{y}_{\gamma}^{2}-\hat{z}_{\gamma}^{2}\right)+2 \hat{y}_{\gamma}^{2}\right)\left(\hat{x}_{\gamma}-\hat{z}_{\gamma}\right) b_{2, \gamma}^{2}-b_{1, \gamma}\left(\hat{x}_{\gamma}^{2}-\hat{y}_{\gamma}^{2}\right)+\hat{x}_{\gamma}
\end{array}\right)}{\Delta_{2}} \\
& v_{\gamma}=\frac{\left(\begin{array}{c}
\left(\hat{x}_{\gamma}-\hat{z}_{\gamma}\right)\left(\hat{x}_{\gamma}+2 \hat{y}_{\gamma}+\hat{z}_{\gamma}\right)\left(\hat{x}_{\gamma}-2 \hat{y}_{\gamma}+\hat{z}_{\gamma}\right) b_{1, \gamma} b_{2, \gamma} \\
-\left(\hat{x}_{\gamma}\left(\hat{x}_{\gamma}+\hat{z}_{\gamma}\right)+2 \hat{y}_{\gamma}^{2}\right) b_{2, \gamma}-\left(\hat{x}_{\gamma}^{2}-\hat{z}_{\gamma}^{2}\right) b_{1, \gamma}+\hat{x}_{\gamma}
\end{array}\right)}{\Delta_{1}}, \\
& u_{\gamma}=\frac{\hat{y}_{\gamma}\left(b_{1, \gamma}\left(\hat{x}_{\gamma}-\hat{z}_{\gamma}\right)-1\right)}{\Delta_{1}}, \\
& t_{\gamma}=\frac{\hat{z}_{\gamma}-\left(\hat{x}_{\gamma} \hat{z}_{\gamma}-\hat{y}_{\gamma}^{2}\right)\left(b_{1, \gamma}+b_{2, \gamma}\right)+b_{1, \gamma} b_{2, \gamma}\left(\hat{z}_{\gamma}\left(\hat{x}_{\gamma}^{2}-\hat{z}_{\gamma}^{2}\right)-2 \hat{y}_{\gamma}^{2}\left(\hat{x}_{\gamma}-\hat{z}_{\gamma}\right)\right)}{\Delta_{2}}
\end{aligned}
$$

where

$$
\begin{aligned}
\Delta_{1} & =b_{1, \gamma} b_{2, \gamma}^{2}\left(\hat{x}_{\gamma}-\hat{z}_{\gamma}\right)\left(\hat{x}_{\gamma} \hat{z}_{\gamma}-4 \hat{y}_{\gamma}^{2}\right)+\left(2 \hat{y}_{\gamma}^{2} b_{1, \gamma}+\hat{x}_{\gamma}\right)\left(b_{1, \gamma}+b_{2, \gamma}\right) \\
& +b_{1, \gamma} b_{2, \gamma}\left(b_{2, \gamma}\left(\hat{x}_{\gamma}^{3}-\hat{z}_{\gamma}^{3}\right)-\left(2 \hat{x}_{\gamma}-\hat{z}_{\gamma}\right)\left(\hat{x}_{\gamma}+\hat{z}_{\gamma}\right)\right)-1, \\
\Delta_{2} & =\left(\left(\hat{x}_{\gamma}-\hat{z}_{\gamma}\right) b_{2, \gamma}-1\right)\left(\left(\left(\hat{x}_{\gamma}-\hat{z}_{\gamma}\right)\left(\hat{x}_{\gamma}+2 \hat{y}_{\gamma}+\hat{z}_{\gamma}\right)\left(\hat{x}_{\gamma}-2 \hat{y}_{\gamma}+\hat{z}_{\gamma}\right)\right.\right. \\
& \left.-\hat{x}_{\gamma}\left(\hat{x}_{\gamma}+\hat{z}_{\gamma}\right)+2 \hat{y}_{\gamma}^{2}\right) b_{2, \gamma}^{2}\left(\left(\left(\hat{z}_{\gamma}\left(\hat{z}_{\gamma}-\hat{x}_{\gamma}\right)-2\left(\hat{x}_{\gamma}^{2}-\hat{y}_{\gamma}^{2}\right)\right) b_{1, \gamma}+2 \hat{x}_{\gamma}\right.\right. \\
& \left.\left.\left.+\hat{z}_{\gamma}\right) b_{2, \gamma}+\hat{x}_{\gamma} b_{1, \gamma}-1\right)\right),
\end{aligned}
$$

with $b_{1, \gamma}=B_{11}^{(n)}$ and $b_{2, \gamma}=B_{1919}^{(n)}=B_{3737}^{(n)}=B_{5555}^{(n)}$.

As the pivotal elements for each of the carpet models have been determined, expressions detailing the electrical impedance and transmission and reception sensitivities for each device can now be calculated. 


\section{BOUNDARY CONDITIONS AND DERIVATION OF TRANSDUCER OPERATING CHARACTERISTICS}

The three transducer models inspired by the Sierpinski carpet have been obtained in a similar manner to the Sierpinski gasket transducer [9]. For each model, mechanical loads are to be placed at the output vertices and an electrical load is to be positioned at the input vertex. For the backing material it is desirable that the attenuation parameter is high so that vibrations at the back are prevented. For that reason it may be assumed that there is only a wave travelling away from the piezoelectric material [9]. Expressions for the displacement in the load and backing material are given as

$$
\bar{u}_{L}=A_{L} \exp \left(\frac{-p v_{T} x_{L}}{\Delta x v_{L}}\right)+B_{L} \exp \left(\frac{p v_{T} x_{L}}{\Delta x v_{L}}\right)
$$

and

$$
\bar{u}_{B}=A_{B} \exp \left(\frac{-p v_{T} x_{B}}{\Delta x v_{B}}\right)
$$

where the subscript $L$ and $B$ refer to the mechanical load and backing layer respectively, $v_{T}$ is the wave velocity in the piezoelectric material and $A_{L}, A_{B}$ and $B_{L}$ are constants that represent the forward and backward traveling waves. Applying the conditions of continuity of displacement at the transducer boundaries (and accounting for symmetries of the Green function matrix) and of force results in

$$
\begin{gathered}
u_{1}=u_{A}=A_{B}, \\
u_{19}=u_{37}=u_{55}=u_{B}=u_{C}=u_{D}=A_{L}+B_{L}, \\
u_{1}-u_{A}-\frac{h Q}{Y_{T} \xi}=-\frac{Z_{B}}{Z_{T}} p A_{B}, \\
u_{B}-u_{19}-\frac{h Q}{Y_{T} \xi}=\frac{Z_{L}}{Z_{T}} p\left(-A_{L}+B_{L}\right),
\end{gathered}
$$

where $Q$ is the electrical charge applied to the transducer at vertex $A$ and passes through in the direction of the electric field, $Z_{B}, Z_{L}$ and $Z_{T}$ are the mechanical impedances of the backing layer, load and transducer respectively and $\xi$ is the ratio of the cross-sectional 
area of each edge to its length. Equations (4.3) and (4.6) are then used to determine the elements of the boundary condition matrix $B^{(n)}$,

$$
B_{i j}=\left\{\begin{array}{cl}
\frac{1}{1-p \frac{Z_{B}}{Z_{T}}} & \text { if } i=j=1 \\
\frac{1}{1-p \frac{Z_{L}}{Z_{T}}} & \text { if } i=j=19,37 \text { or } 55 \\
0 & \text { otherwise }
\end{array}\right.
$$

and the vector $\underline{c}^{(n)}$

$$
c_{i}=\left\{\begin{array}{cl}
-\frac{h Q}{Y_{T} \xi}\left(\frac{1}{\left.1-p \frac{Z_{B}}{Z_{T}}\right)}\right. & \text { if } i=1 \\
\left(\frac{h Q}{Y_{T} \xi}-2 p A_{L} \frac{Z_{L}}{Z_{T}}\right)\left(\frac{1}{\left.1-p \frac{Z_{L}}{Z_{T}}\right)}\right. & \text { if } i=19,37 \text { or } 55 . \\
0 & \text { otherwise }
\end{array}\right.
$$

The derivations in Eqs. (4.7) and (4.8) will be used to determine the expressions for the electrical impedance and transmission and reception sensitivities for the three devices.

An expression for the non-dimensionalized electrical impedance is given by

$$
\hat{Z}_{E, i}=\frac{Z_{T}}{p C_{0} Y_{T} \xi Z_{0}}\left(1+\frac{h^{2} C_{0}\left(\alpha_{i}+\beta_{i}\right)}{Y_{T} \xi}-\frac{2 h C_{0} p \alpha_{i} A_{L} Z_{L}}{Q Z_{T}}\right) \text { for } i=\eta, \delta, \gamma
$$

where $C_{0}$ is the capacitance and

$$
\begin{gathered}
\alpha_{\eta}=\frac{z_{\eta}-v_{\eta}}{1-p \frac{Z_{L}}{Z_{T}}}, \quad \beta_{\eta}=\frac{z_{\eta}-x_{\eta}}{1-p \frac{Z_{B}}{Z_{T}}}, \\
\alpha_{\delta}=\frac{2 y_{\delta}-w_{\delta}-t_{\delta}}{1-p \frac{Z_{L}}{Z_{T}}}, \quad \beta_{\delta}=\frac{y_{\delta}-x_{\delta}}{1-p \frac{Z_{B}}{Z_{T}}}, \\
\alpha_{\gamma}=\frac{2 y_{\gamma}+z_{\gamma}-w_{\gamma}-u_{\gamma}-t_{\gamma}}{1-p \frac{Z_{L}}{Z_{T}}} \quad \text { and } \quad \beta_{\gamma}=\frac{y_{\gamma}-x_{\gamma}}{1-p \frac{Z_{B}}{Z_{T}}} .
\end{gathered}
$$

Now that the electrical impedances for each of the transducer models have been calculated, these analytical expressions are used to derive the necessary transmission and reception sensitivities for each device. In transmission mode, the conversion of electrical signal to mechanical vibration is achieved from the application of a voltage [25-27]. 
When the transducer is transmitting there is no force incident at the front face of the transducer, and so there is no forward traveling wave in the load, i.e. $A_{L}=0[9]$. Thus, the non-dimensionalized transmission sensitivity is given by

$$
\psi_{i}=\frac{F}{V h C_{0}}=\frac{a Z_{T} K_{i}^{(n)}}{Y_{T} \xi C_{0}\left(Z_{E, i}+b\right)} \text { for } i=\eta, \delta, \gamma,
$$

where

$$
\begin{aligned}
& K_{\eta}^{(n)}=\left(\frac{1}{1-p \frac{Z_{L}}{Z_{T}}}\right)\left(1+\frac{v_{\eta}}{1-p \frac{Z_{L}}{Z_{T}}}-\frac{z_{\eta}}{1-p \frac{Z_{B}}{Z_{T}}}\right), \\
& K_{\delta}^{(n)}=\left(\frac{1}{1-p \frac{Z_{L}}{Z_{T}}}\right)\left(1+\frac{w_{\delta}+t_{\delta}}{1-p \frac{Z_{L}}{Z_{T}}}-\frac{y_{\delta}}{1-p \frac{Z_{B}}{Z_{T}}}\right),
\end{aligned}
$$

and

$$
K_{\gamma}^{(n)}=\left(\frac{1}{1-p \frac{Z_{L}}{Z_{T}}}\right)\left(1+\frac{w_{\gamma}+u_{\gamma}+t_{\gamma}}{1-p \frac{Z_{L}}{Z_{T}}}-\frac{y_{\gamma}}{1-p \frac{Z_{B}}{Z_{T}}}\right) .
$$

In reception mode, the piezoelectric material converts mechanical energy into electrical energy through the contact of sound waves resulting in the production of an electrical signal [25-27]. The front face of the transducer will be subjected to external forces when in receiving mode, hence $A_{L} \neq 0$. The expression for amplitude of the forward propagating wave is given by $A_{L}=-\frac{F v_{L}}{p Y_{L} \xi v_{T}}$. Hence, the non-dimensionalized reception sensitivity is

$$
\phi_{i}=\frac{V h C_{0}}{F}=\frac{2 h^{2} C_{0} \alpha_{i}}{Y_{T} \xi\left(1-\frac{a Z_{T}}{p C_{0} Y_{T} \xi\left(Z_{E, i}+b\right)}\left(1+\frac{h^{2} C_{0}\left(\alpha_{i}+\beta_{i}\right)}{Y_{T} \xi}\right)\right)} \text { for } i=\eta, \delta, \gamma
$$

With the relevant operating transducer characteristics now derived, computer simulation models can be utilized in order to determine the possible benefits of a transducer based on the design of the Sierpinski carpet. The electrical impedance, transmission and reception sensitivities for each of the models are presented in the following section. 


\section{COMPARISON AND EFFECTIVENESS OF STANDARD AND PRE-FRACTAL TRANSDUCERS}

To minimise time and costs involved in manufacturing new ultrasonic transducers, mathematical computer models are employed to test the performances of hypothetical designs. These models can give an insight as to what benefit, if any, can be achieved from altering current designs or can suggest the most effective configuration of new designs. In regards to a Sierpinski carpet-like transducer it is only realistically viable to consider the lower generation levels when it comes to potential manufacture. Incrementing the fractal generation level increases the complexity of the transducer model, and it is for this reason that constructing higher generation levels would currently prove to be infeasible. The work presented in this paper allows for comparison of the three Sierpinski carpet models with the Sierpinski triangle, each at generation level three, and the currently used Euclidean transducer.

\begin{tabular}{|c||c|c|c|}
\hline & Symbol & Magnitude & Dimensions \\
\hline \hline Elastic constant & $c_{33}$ & $11.74 \times 10^{10}$ & $\mathrm{~N} \mathrm{~m}^{-2}$ \\
\hline Piezoelectric stress coefficient & $e_{33}$ & 23.3 & $\mathrm{C} \mathrm{m}^{-2}$ \\
\hline Dielectric constant & $\epsilon_{33}$ & $1.47 \times 10^{3}$ & - \\
\hline Density & $\rho_{T}$ & $7.5 \times 10^{3}$ & $\mathrm{~kg} \mathrm{~m}^{-3}$ \\
\hline Parallel electrical load & $Z_{P}$ & $10^{3}$ & $\Omega$ \\
\hline Series electrical load & $Z_{0}$ & 50 & $\Omega$ \\
\hline
\end{tabular}

Table 1: Transducer material parameters [28].

Each of the transducers, fractal and Euclidean, are modelled on a PZT-5H ceramic [28]. The material parameters used to derive the expressions for the transducers operating characteristics corresponding are shown in Table 1. Figure 6 shows the comparison for the electrical impedance between the three Sierpinski carpet models $\left(\hat{Z}_{E, \eta}\right.$, $\left.\hat{Z}_{E, \delta}, \hat{Z}_{E, \gamma}\right)$, the Sierpinski gasket $\left(\hat{Z}_{E, G}\right)$ and Euclidean $\left(\hat{Z}_{E, E}\right)$ transducers. Electrical impedance profiles are important as they allow us to determine the potential efficiency of the theoretical transducer. The features of interest from these plots are the occurrence of the electrical and mechanical resonant frequencies. The electrical resonant frequency $f_{e}$ and the mechanical resonant frequency $f_{m}$ are determined by locating the first minimum and first maximum in the plot respectively [14, 29]. In Fig. 6 all devices show 


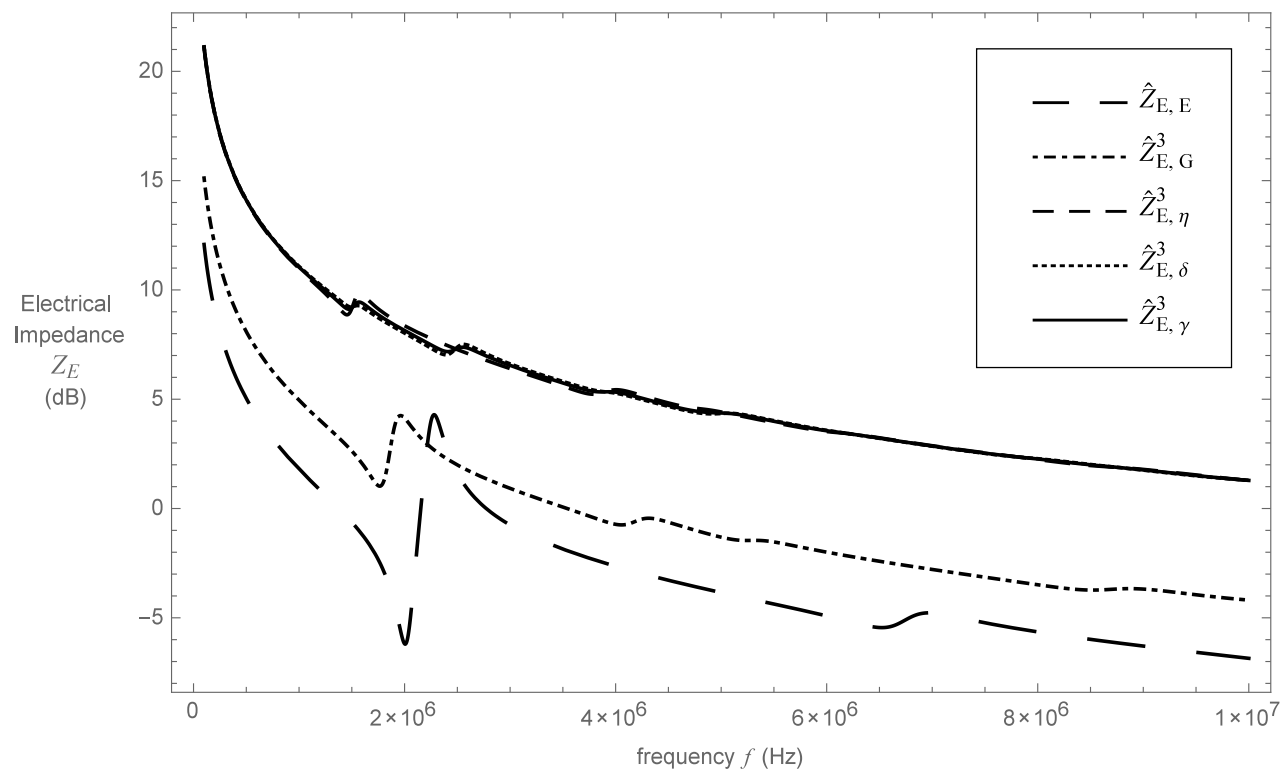

Figure 6: Non-dimensionalised electrical impedances $\hat{Z}_{E}(\mathrm{~dB})$ versus frequency $f(\mathrm{~Hz})$ for the three theoretical Sierpinski carpet transducers and the Sierpinski gasket transducer at fractal generation level $n=3$ and the Euclidean transducer.

qualitatively similar results and adhere to similar patterns with the carpet devices resonating at higher electrical impedances. This most likely relates to the size of the length scales at this fractal generation level. In this instance, the length scales within the carpet's internal structure would be considerably smaller than the ones present within the gasket and standard devices. This would consequently result in higher values for the electrical impedance. The electrical and mechanical resonant frequencies together with the corresponding electrical impedances for each device are given in Table 2.

\begin{tabular}{|c||c|c||c|c|}
\hline & $f_{e}$ & $Z_{E}$ & $f_{m}$ & $Z_{E}$ \\
\hline \hline Euclidean $E$ & 1.901 & -6.172 & 2.181 & 4.291 \\
\hline Sierpinski Gasket $G$ & 1.677 & 1.059 & 1.863 & 4.251 \\
\hline Carpet Model $\eta$ & 1.355 & 8.827 & 1.476 & 9.823 \\
\hline Carpet Model $\delta$ & 2.302 & 6.969 & 2.463 & 7.506 \\
\hline Carpet Model $\gamma$ & 1.374 & 9.072 & 1.470 & 9.445 \\
\hline
\end{tabular}

Table 2: Device electrical and mechanical resonant frequencies at fractal generation level $n=3$.

The receiving and transmitting characteristics for each device were evaluated by determining the three metrics, maximum amplitude (gain $g$ ), 3-dB bandwidth $(B W)$ and the gain bandwidth product $(G B P)$. This is simply the product of the maximum 
amplitude and bandwidth. High amplitude is a desirable attribute since this relates to the production of energy; the higher the amplitude the greater the generation of energy. The $3-\mathrm{dB}$ bandwidth of the device is often used as the cut-off frequency since this gives the range of frequencies over which the transducer operates efficiently. The gain bandwidth product was used as the principle figure of merit to establish the optimal device. One important feature of this metric is that it can serve as estimation for the maximum bandwidth at the maximum amplitude.

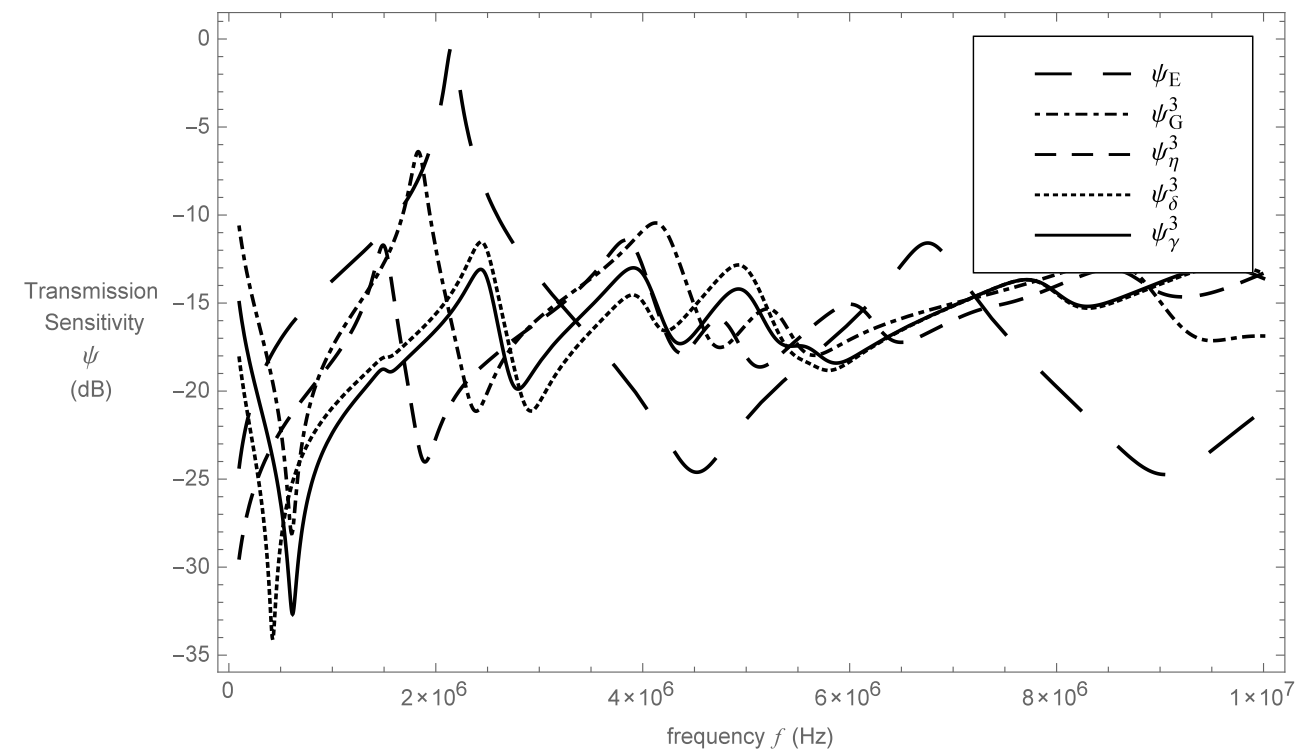

Figure 7: Non-dimensionalised transmission sensitivities $\psi(\mathrm{dB})$ versus frequency $f(\mathrm{~Hz})$ for the three theoretical Sierpinski carpet transducers and the Sierpinski gasket transducer at fractal generation level $n=3$ and the Euclidean transducer.

Comparison of transmission sensitives are shown in Fig. 7. From these results it is evident that the transmission sensitivity for the Euclidean device has the highest amplitude and is greater by $12.628 \mathrm{~dB}$ than model $\gamma$ of the carpet device, which offers the lowest. Consequently, this has resulted in a reduced bandwidth of $0.187 \mathrm{MHz}$ for the Euclidean device leading to a percentage bandwidth of $9 \%$, while the carpet model $\gamma$ has a percentage bandwidth of $22 \%$. Carpet models $\eta$ and $\delta$ follow very closely with a percentage bandwidth each of $20 \%$, which compares to just $13 \%$ for the Sierpinski gasket device. The results obtained for the three carpet models, represent a substantial improvement on device performance over both the currently used Euclidean transducer and the Sierpinski gasket prototype. There are however, concerns over the values of the 
principle figure of merit, as they are much lower than the value for the Euclidean device. This is most likely a result of the low amplitudes since the gain bandwidth product is calculated using $G B P=g \times B W$, where the calculation is performed using the value of the amplitude before its conversion into decibels. Table 3 presents the metrics describing the transmission characteristics for each transducer.

\begin{tabular}{|c||c|c|c|}
\hline \multicolumn{1}{|c||}{} & \multicolumn{3}{c|}{ Metrics } \\
\cline { 2 - 4 } & $\begin{array}{c}\text { Gain } \\
(g) \mathrm{dB}\end{array}$ & $\begin{array}{c}\text { 3-dB Bandwidth } \\
(B W) \mathrm{MHz}\end{array}$ & $\begin{array}{c}\text { Gain Bandwidth } \\
\text { Product }(G B P)\end{array}$ \\
\hline \hline Euclidean $E$ & -0.371 & 0.187 & 0.172 \\
\hline Sierpinski Gasket $G$ & -6.399 & 0.221 & 0.051 \\
\hline Carpet Model $\eta$ & -11.387 & 0.735 & 0.053 \\
\hline Carpet Model $\delta$ & -11.534 & 0.461 & 0.032 \\
\hline Carpet Model $\gamma$ & -12.999 & 0.837 & 0.042 \\
\hline
\end{tabular}

Table 3: The transmission response of the Euclidean and fractal transducers at generation level $n=3$.

The figures of merit for each transducer in receiving mode were calculated and the results are presented in Table 4. Comparison between the fractal and Euclidean transducers, illustrated in Fig. 8 shows a good match with more resonances in the carpet devices. This is to be expected as the carpet devices feature more complexity in their structure, resulting in a wider range of length scales. In terms of device bandwidth, all carpet models outperform the Euclidean and gasket transducers. In particular models $\delta$ and $\gamma$ more than double the operational bandwidth of the gasket and Euclidean devices, indicating that these models are more efficient at operating over a larger range of frequencies when acting as a receiver of ultrasound. Furthermore, there is a significant improvement in regards to the principal figure of merit in all three carpet models. This presents an increase of at least $38 \%$ compared to the Euclidean device and an increase of at least $82 \%$ compared to the gasket device. The results suggest strongly that it would be worthwhile for a prototype based on the Sierpinski carpet to be built, to determine whether experimental results corroborate these theoretical results.

Figure 9 illustrates the transmission response gain bandwidth product as a function of frequency for the three carpet models. By observing the behaviour of each device it is clear that the greatest gain bandwidth product is present in model $\eta$ at a frequency of $3.737 \mathrm{MHz}$, which corresponds to the model's peak amplitude; see Table 3. Evidence 


\begin{tabular}{|c||c|c|c|}
\hline \multicolumn{1}{|c||}{} & \multicolumn{3}{c|}{ Metrics } \\
\cline { 2 - 4 } & $\begin{array}{c}\text { Gain } \\
(g) \mathrm{dB}\end{array}$ & $\begin{array}{c}\text { 3-dB Bandwidth } \\
(B W) \mathrm{MHz}\end{array}$ & $\begin{array}{c}\text { Gain Bandwidth } \\
\text { Product }(G B P)\end{array}$ \\
\hline \hline Euclidean & 3.270 & 0.259 & 0.549 \\
\hline Sierpinski Gasket & 2.751 & 0.221 & 0.417 \\
\hline Carpet Model $\eta$ & 4.629 & 0.261 & 0.757 \\
\hline Carpet Model $\delta$ & 1.615 & 0.533 & 0.774 \\
\hline Carpet Model $\gamma$ & 1.674 & 0.539 & 0.792 \\
\hline
\end{tabular}

Table 4: The receiving response of the Euclidean and fractal transducers at generation level $n=3$.

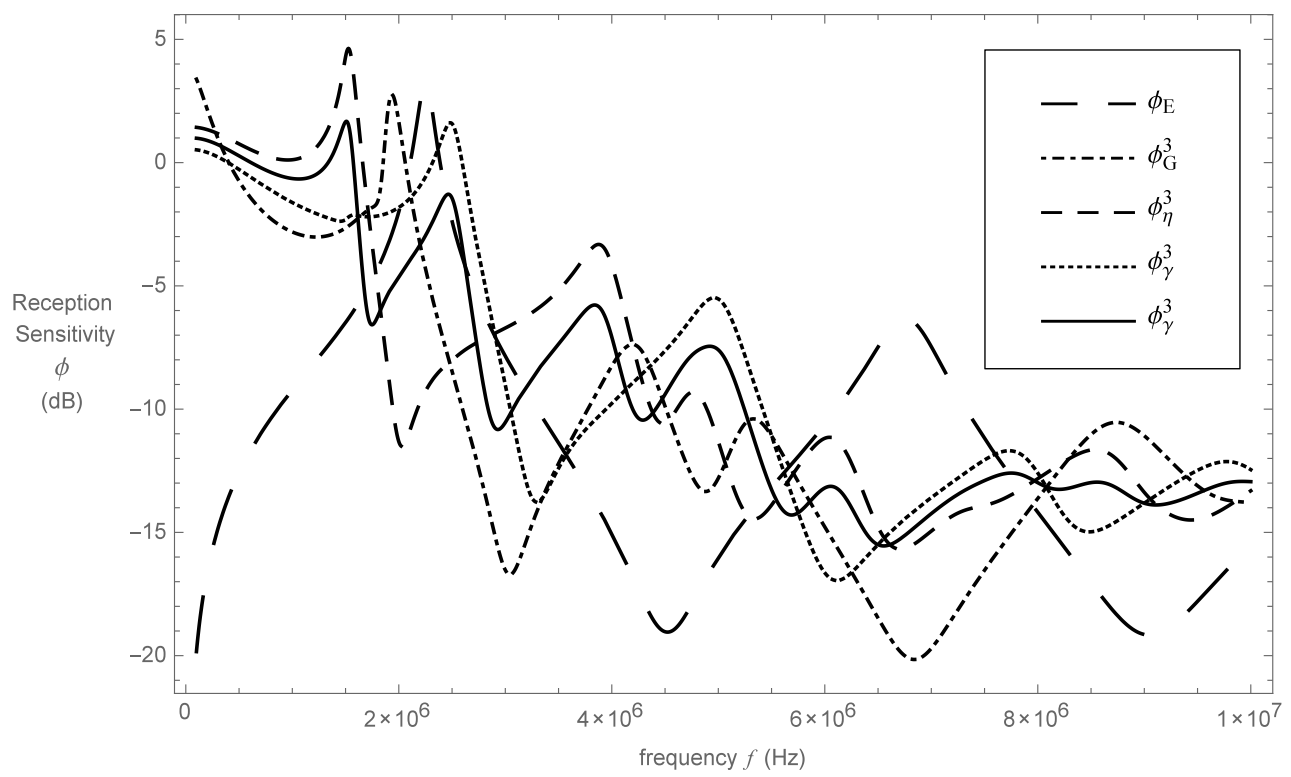

Figure 8: Non-dimensionalised reception sensitivities $\phi(\mathrm{dB})$ versus frequency $f(\mathrm{~Hz})$ for the three theoretical Sierpinski carpet transducers and the Sierpinski gasket transducer at fractal generation level $n=3$ and the Euclidean transducer.

would suggest through the data analysed that model $\gamma$ provides the greatest value for the gain bandwidth product in reception mode at its peak amplitude frequency and that the value of this metric decreases at higher frequencies. This trend is also reflected in models $\eta$ and $\delta$, in that the greatest gain bandwidth product value occurs at lower frequencies which correspond to the maximum amplitudes. These results are illustrated in Fig. 10. 


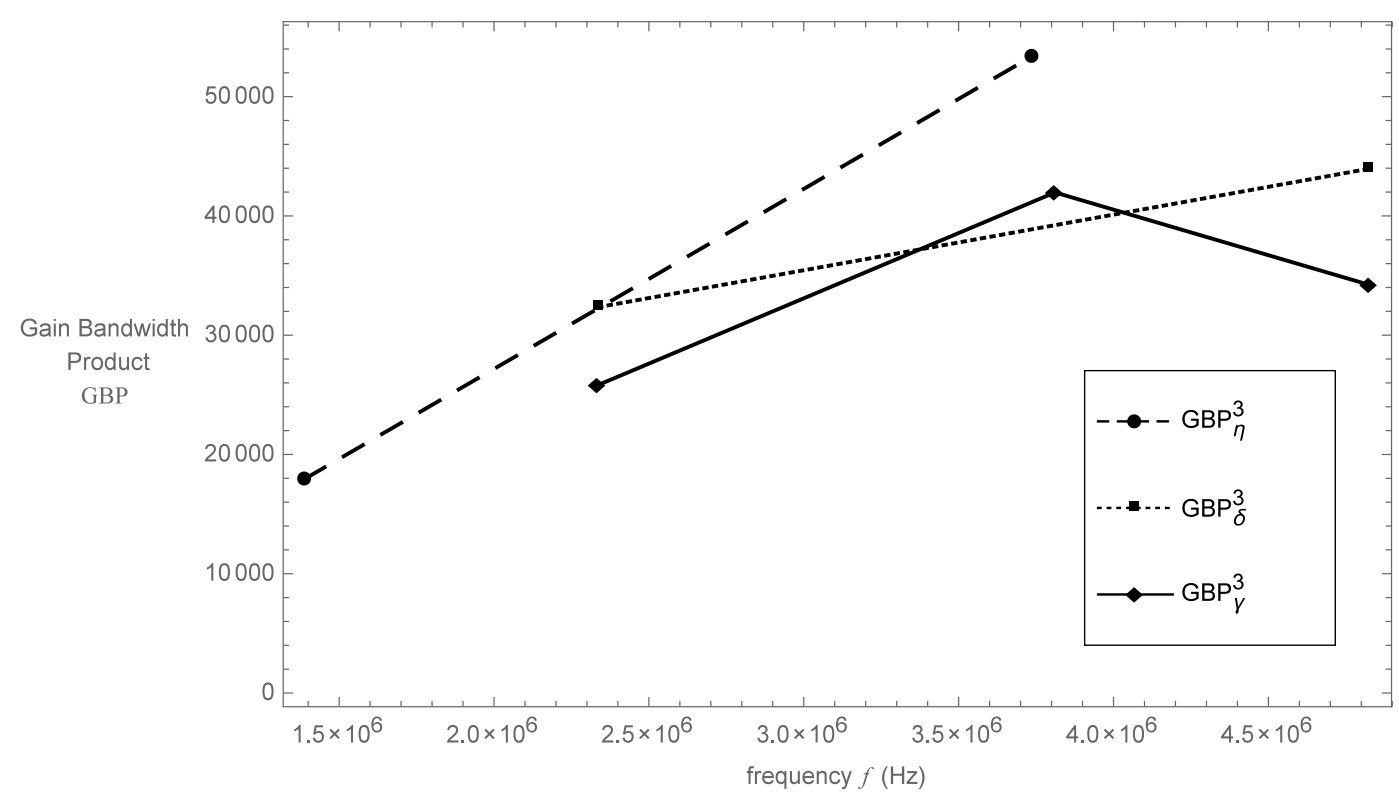

Figure 9: The gain bandwidth product for transmission sensitivity $\psi$ for the three Sierpinski carpet models versus frequency $f(\mathrm{~Hz})$ at fractal generation level $n=3$.

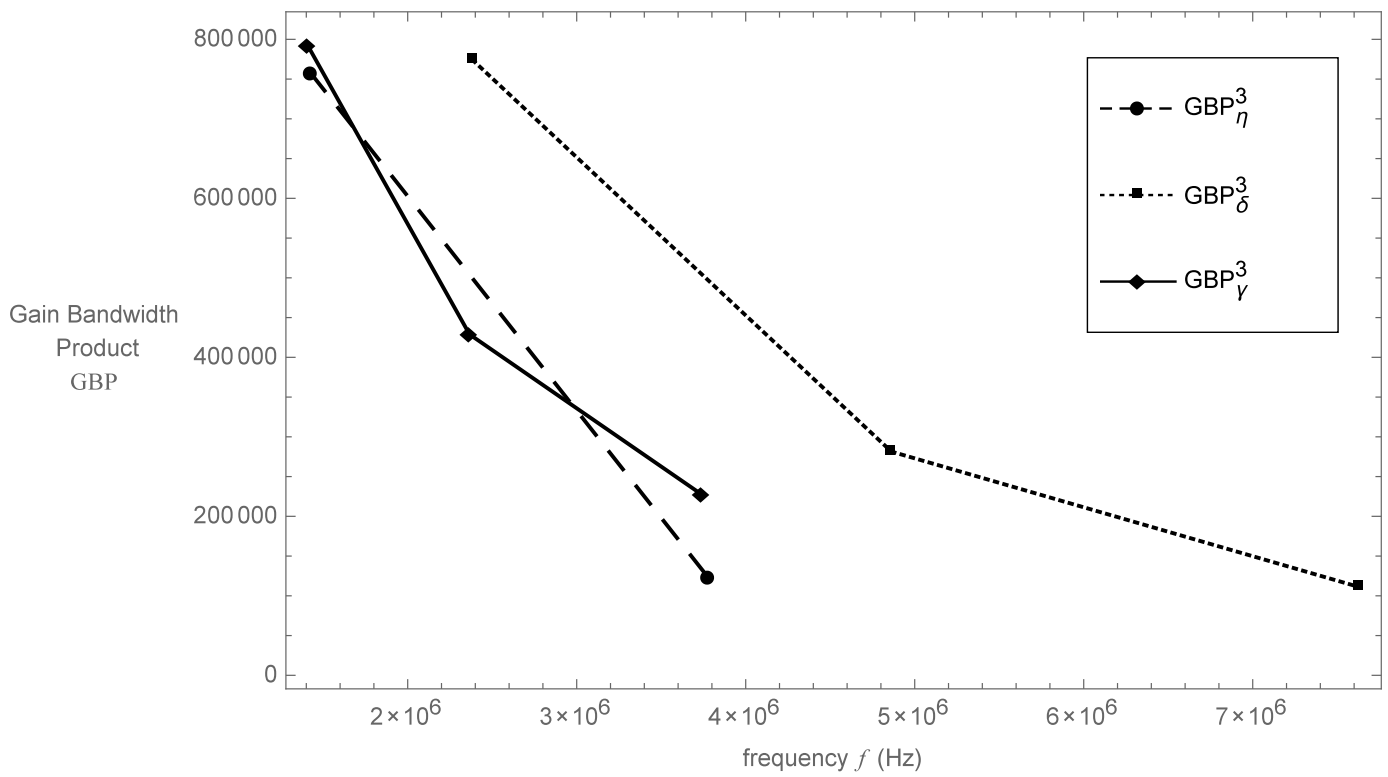

Figure 10: The gain bandwidth product for reception sensitivity $\phi$ for the three Sierpinski carpet models versus frequency $f(\mathrm{~Hz})$ at fractal generation level $n=3$.

\section{CONCLUSIONS AND DISCUSSION}

The analysis presented in this paper extends earlier research of implementing fractal geometry into ultrasonic transducer design $[9,12-14,30,31]$. A renormalization adap- 
tation was used to investigate the potential performance of a fractal like transducer. The fractal used as inspiration for the transducer design was the Sierpinski carpet. The non-constant coordination number of the carpet pre-fractals necessitated a more computationally demanding approach to produce the Green function recurrent relations than was employed in previous papers. The Sierpinski carpet was examined by constructing three individual transducer models all influenced by its design. The variations in models corresponded to the number of the boundary conditions and for each of the models a lattice counterpart was used to analyse the transducers operating characteristics. Although all three models are relatively similar in their form, the symmetries of the lattice for model $\delta$ resulted in the need for additional independent Green functions.

Due to the limitations of the computing power available that were used to produce the results for the theoretical Sierpinski carpet-like transducer, only the first three fractal generation levels were investigated. High performance computers could be used as an alternative to yield the operational characteristics at higher fractal generation levels. However for manufacturing purposes, current restrictions would limit the construction of pre-fractal transducers beyond generation level three. In the instance of the Sierpinski gasket transducer, a prototype based on the design of its fourth generation level was constructed [13]. The Sierpinski gasket is of a simpler design than the Sierpinski carpet and so the fabrication of a Sierpinski carpet prototype at the same generation level may currently prove to be unfeasible. This paper investigated a fractal inspired transducer using the Green function renormalization technique as a method for obtaining the transducer operating characteristics. For a comparison it would be beneficial to build another model of a transducer based on the carpet structure using finite element analysis. Such research is currently underway on the Sierpinski carpet pre-fractal [32] and has already been performed for the Sierpinski gasket pre-fractal [14].

To assess the performance of each transducer the electrical impedance, and transmission and reception sensitivities were plotted as a function of operating frequency. These were then compared to a previously investigated Sierpinski gasket transducer as well as to a standard Euclidean design transducer which is commonly used in industry. As expected it was the carpet devices which experienced more resonance at a wider 
range of frequencies. In regards to the transmission sensitivities, an increase in the fractal generation level resulted in an increase in the device's bandwidth. This increase in bandwidth however, has led to a reduction in the device's amplitude. From the results it was shown that the each carpet model outperformed both the gasket and Euclidean transducers in terms of its bandwidth. However, the greatest amplitude and gain bandwidth product were present in the Euclidean transducer. While not explicitly shown in this paper, in reception mode an increase in fractal generation level results in an increase in both amplitude and bandwidth. Furthermore, the carpet devices surpassed the gasket and Euclidean devices in regards to bandwidth and gain bandwidth product, with carpet model $\eta$ outperforming the gasket and Euclidean transducers in all three metrics. The principal figure of merit that was used to determine the most efficient transducer was the gain bandwidth product. The three carpet models were then compared to one another in regards to their transmitting and receiving capabilities, using this figure of merit. Thus in transmission mode, it was model $\eta$ which presented the best result at its peak amplitude frequency. In reception mode, model $\gamma$ had overall the highest value. The 3-dB bandwidth indicates that model $\gamma$ outperforms all devices in both transmission and reception. This suggests that model $\gamma$ would be suitable in ultrasonic design to enhance current transducer performance, especially when considering the results in reception mode. In receiving mode as the fractal generation level increases, so does the principle figure of merit for all the carpet models. This was to be expected since the range of length scales increased with the fractal generation level. Similarly, the same could be said in transmitting mode, although with a few discrepancies.

Looking ahead, it may be possible to construct a system capable of determining the coordination number for each vertex in the sequence of lattices. This would enable the application of the Green function renormalization in a similar manner as used for the Sierpinski gasket, employing the recurrent relation equation (Eq. 2.8) to extract the next generation level pivotal Green functions. Hence the need to obtain the inverse matrices at each fractal generation level would be removed, reducing considerable computational cost. Another area of interest would be in transducer performance optimization akin to that done for the Sierpinski gasket pre-fractal [33]. A technique for increasing the 
values for each of the figures of merit could be achieved by sampling different material parameters. This may assist in establishing the optimal material that could be used for ultrasonic transducers. Furthermore, investigating the inverse problem of finding material and design parameters which produce a desired output would also be beneficial. Such inverse problems would require the consideration of the well-posedness of the model [34-37].

This research is concerned with increasing the operational bandwidth of piezoelectric ultrasonic transducers by incorporating pre-fractal geometries into their design. These geometries were of interest as they span a range of length scales. More fractal inspired transducers may be designed by implementing other fractal structures or through the modification of these structures. Specifically modified Sierpinski carpets could realise better operating characteristics. Research on transducer performance designed on a modified Sierpinski gasket has already begun [31]. The purpose of adjusting such fractal designs would be to yield wider length scaled devices.

\section{ACKNOWLEDGEMENT}

This work has been supported by a Centenary Doctoral Scholarship from the University of South Wales and is gratefully acknowledged.

\section{NOMENCLATURE}

$\begin{array}{cc}A, B, C, D & \text { Fictitious vertices } \\ A_{L}, A_{B} & \text { Forward traveling waves within the load and backing layer } \\ A^{(n)} & \text { Discretized Laplacian matrix } \\ B_{L}, B_{B} & \text { Backward traveling waves within the load and backing layer } \\ B^{(n)} & \text { Boundary conditions matrix } \\ B W & \text { B-dB bandwidth } \\ \underline{c}^{(n)} & \text { Boundary condition vector } \\ C_{0} & \text { Capacitance } \\ D & \text { Electric displacement } \\ E & \text { Electric field }\end{array}$




\begin{tabular}{|c|c|}
\hline$f$ & Frequency \\
\hline$F$ & Force \\
\hline$f_{e}, f_{m}$ & Electrical and mechanical resonant frequencies \\
\hline$g$ & Maximum amplitude \\
\hline$G B P$ & Gain bandwidth product \\
\hline$G^{(n)}$ & Green function matrix \\
\hline$\hat{G}^{(n)}$ & Bare Green function matrix \\
\hline$h$ & Piezoelectric constant \\
\hline$H^{(n)}$ & Adjacency matrix \\
\hline $\bar{H}^{(n)}$ & Block diagonal matrix whose $v$ blocks equal $H^{(n)}$ \\
\hline$I_{n}$ & $n \times n$ identity matrix \\
\hline$l$ & Transducer thickness \\
\hline$L$ & Lattice side length \\
\hline$n$ & Fractal generation level \\
\hline$N_{n}$ & Total number of vertices \\
\hline$p$ & Laplace transform variable \\
\hline$q$ & coordination number \\
\hline$Q$ & Electrical charge \\
\hline$S$ & Strain \\
\hline$T$ & Stress \\
\hline$u$ & Particle displacement \\
\hline$V$ & Voltage \\
\hline$v_{T}, v_{L}, v_{B}$ & Wave velocity in piezoelectric material, load and backing layer \\
\hline$V^{(n)}$ & Sparse matrix representing the connectivity of sub-graphs \\
\hline$Y$ & Young's modulus \\
\hline$Z_{B}, Z_{L}, Z_{T}$ & Mechanical impedance of backing layer, load and transducer \\
\hline$\hat{Z}_{E}$ & Non-dimensionalized electrical impedance \\
\hline$Z_{0}$ & Series electrical load \\
\hline$\eta, \delta, \gamma$ & Sierpinski carpet models \\
\hline$\Delta x$ & Distance between neighboring vertices \\
\hline
\end{tabular}


$\xi \quad$ Ratio of the cross-sectional area and edge length

$\rho$

Density

$\psi \quad$ Non-dimensionalized transmission sensitivity

\section{REFERENCES}

[1] S. N. Ramadas and G. Hayward, A knowledge based approach for design optimization of ultrasonic transducers and arrays, Paper presented at IEEE International Ultrasonics Symposium, 2247-2250, Rotterdam, The Netherlands, 2005.

[2] R. H. Warring and S. Gibilisco, Fundamentals of Transducers, ed. (Tab Books, Pennsylvania, 1985).

[3] D. W. Schindel, D. A. Hutchins, L. Zou and M. Sayer, The design and characterization of micro-machined air-coupled capacitance transducers, IEEE Trans. Ultrason. Ferroelectr. Freq. Control. 42(1995) 42-50.

[4] R. A. Nobel, A. D. R. Jones, T. J. Robertson, D. A. Hitchins and D. R. Billson, Novel wide bandwidth, micromachined ultrasonic transducers, IEEE Trans. Ultrason. Ferroelectr. Freq. Control. 48(2001) 1495-1507.

[5] A. J. Walker, A. J. Mulholland, E. Campbell and G. Hayward, A theoretical model of a new electrostatic transducer incorporating fluidic amplification, Paper presented at IEEE International Ultrasonics Symposium, 1409-1412, Beijing, China, 2008.

[6] A. J. Walker and A. J. Mulholland, A theoretical model of an electrostatic ultrasonic transducer incoporating resonating conduits, IMA. J. Appl. Math. 75(2010) 769810.

[7] A. J. Walker and A. J. Mulholland, A theoretical model of an ultrasonic transducer incorporating spherical resonators, IMA. J. Appl. Math. 81(1) (2016) 1-25.

[8] B. Siciliano and O. Khatib, Springer Handbook of Robotics, ed. (Springer Science and Business Media, Verlag Berlin Heidelberg, 2008). 
[9] A. J. Mulholland and A. J. Walker, Piezoelectric ultrasonic transducers with fractal geometry, Fractals. 19(4) (2011) 469-479.

[10] J. A. Flint, A biomimetic antenna in the shape of a bat's ear, IEEE Antennas Wireless Propag. Lett. 5(1) (2006) 145-147.

[11] D. Robert and M. C. Göpfert, Novel schemes for hearing and orientation in insects, Curr. Opin. Neurobiol. 12 (2002) 715-720.

[12] L.-A. Orr, A. J. Mulholland, R. L. O'Leary and G. Hayward, Analysis of ultrasonic transducers with fractal architecture, Fractals. 16(4) (2008) 333-349.

[13] A. J. Mulholland, J. W. Mackersie, R. L. O'Leary, A. Gachagan, A. J. Walker and S. N. Ramadas, The use of fractal geometry in the design of piezoelectric ultrasonic transducers, Paper presented at IEEE International Ultrasonics Symposium, 15591562, Orlando, Florida, United States, 2011.

[14] E. A. Algehyne and A. J. Mulholland, A Finite Element Approach to Modelling Fractal Ultrasonic Transducers, IMA. J. Appl. Math. 80 (2015) 1684-1702.

[15] K. Falconer, Techniques in Fractal Geometry, 2nd ed. (Wiley, Chichester, 1997).

[16] K. Falconer, Fractal Geometry: Mathematical Foundations and Applications, 2nd ed. (Wiley, Chichester, 2003).

[17] L. Seuront, Fractals and Multifractals in Ecology and Aquatic Science, (CRC Press, Florida, 2009).

[18] T. G. Dewey, Fractals in Molecular Biophysics, (Oxford University Press, New York, 1998).

[19] M. Giona, W. A. Schwalm, A. Adrover and M. K. Schwalm, Analysis of linear transport phenomena on fractals, Chem. Eng. J. 64 (1996) 45-61.

[20] M. Giona, W. A. Schwalm, M. K. Schwalm and A. Adrover, Exact solution of linear transport equations in fractal media - I. Renormalization analysis and general theory, Chem. Eng. Sci. 51 (1996) 4717-4729. 
[21] A. J. Mulholland, Bounds on the Hausdorff dimension of a renormalisation map arising from an excitable reaction-diffusion system on a fractal lattice, Chaos. Soliton. Fract. 35(2) (2008) 274-284.

[22] W. A. Schwalm and M. K. Schwalm, Extension theory for lattice Green functions, Phys. Rev. B. 37(16) (1988) 9524-9542.

[23] M. K. Schwalm, M. Giona, W. A. Schwalm, A. Adrover and M. Giustiniani, Renormalization analysis and adsorption on fractals and disordered lattices in the presence of energetic disorder, Langmuir. 13(5) (1997) 1128-1137.

[24] M. Giona, Transport phenomena in fractal and heterogeneous media - Input/ Output renormalisation and exact results, Chaos. Soliton. Fract. 7(1996) 13711396.

[25] P. R. Hoskins, K. Martin and A. Thrush, Diagnostic Ultrasound: Physics and Equipment, 2nd ed. (Cambridge University Press, Cambridge, 2010).

[26] K. Nakamura, Ultrasonic Transducers: Materials and Design for Sensors, Actuators and Medical Applications, (Woodhead Publishing, Cambridge, 2012).

[27] M. Schwartz, Smart Materials, (CRC Press, Florida, 2008).

[28] J. Yang, Analysis of Piezoelectric Devices, (World Scientific, Singapore, 2006).

[29] L.-A. Orr, A. J. Mulholland, R. L. O'Leary, A. Parr, R. A. Pethrick and G. Hayward, Theoretical Modelling of Frequency Dependent Elastic Loss in Composite Piezoelectric Transducers, Ultrasonics. 47(1) (2007) 130-137.

[30] S. Canning, A. J. Walker and P. A. Roach, A Mathematical Model of a Novel 3D Fractal-Inspired Piezoelectric Ultrasonic Transducer, Sensors. 16 (2016) 2170 (16 pages).

[31] E. A. Algehyne and A. J. Mulholland, Renormalization analysis of a composite ultrasonic transducer with a fractal architecture, Fractals. 25(2) (2017) 1750015 (14 pages). 
[32] A. J. Mulholland, Investigating a Sierpinski carpet pre-fractal transducer using finite element analysis, University of Strathclyde, IN. 2016 (private communications).

[33] E. Barlow, A. Algehyne and A. J. Mulholland, Investigating the performance of a fractal ultrasonic transducer under varying system conditions, Symmetry. 8(2016) 43 (30 pages).

[34] A. J. Mulholland, R. Picard, S. Trostorff and M. Waurick, On well-posedness for some thermo-piezoelectric coupling models, Math. Meth. Appl. Sci. (2016).

[35] B. Kaltenbacher, T. Lahmer, M. Mohr and M. Kaltenbacher, PDE based determination of piezoelectric material tensors, Eur. J. Appl. Math. 17(4) (2006) 383-416.

[36] T. Lahmer and B. Kaltenbacher, Optimal measurement selection for piezoelectric material tensor identification, Inverse Probl. Sci. Eng. 16(3) (2008) 369-387.

[37] S. N. Chandler-Wilde and D. P. Hewett, Well-posed PDE and integral equation formulations for scattering by fractal screens, Submitted for publication, IN. 2016 (Preprint at arXiv:1611.0953).

\section{APPENDIX A}

\section{Derivation of Green Function Elements}

To illustrate the recursive nature of the fractal graphs (Eq. (2.1)), the adjacency matrix for the first generation level of the Sierpinski carpet lattice is given by

$$
H^{(1)}=\left(\begin{array}{llllllll}
0 & 1 & 0 & 0 & 0 & 0 & 0 & 1 \\
1 & 0 & 1 & 0 & 0 & 0 & 0 & 0 \\
0 & 1 & 0 & 1 & 0 & 0 & 0 & 0 \\
0 & 0 & 1 & 0 & 1 & 0 & 0 & 0 \\
0 & 0 & 0 & 1 & 0 & 1 & 0 & 0 \\
0 & 0 & 0 & 0 & 1 & 0 & 1 & 0 \\
0 & 0 & 0 & 0 & 0 & 1 & 0 & 1 \\
1 & 0 & 0 & 0 & 0 & 0 & 1 & 0
\end{array}\right)
$$

The Green function matrix, as described in Eq. (2.7), is then computed and the pivotal Green functions are extracted from the inverse matrix. As an example, the application 
of Eq. (2.7) on model $\gamma$ results in

$$
\hat{G}^{(1)}=\left(\begin{array}{cccccccc}
3+p^{2} & -1 & 0 & 0 & 0 & 0 & 0 & -1 \\
-1 & 2+p^{2} & -1 & 0 & 0 & 0 & 0 & 0 \\
0 & -1 & 3+p^{2} & -1 & 0 & 0 & 0 & 0 \\
0 & 0 & -1 & 2+p^{2} & -1 & 0 & 0 & 0 \\
0 & 0 & 0 & -1 & 3+p^{2} & -1 & 0 & 0 \\
0 & 0 & 0 & 0 & -1 & 2+p^{2} & -1 & 0 \\
0 & 0 & 0 & 0 & 0 & -1 & 3+p^{2} & -1 \\
-1 & 0 & 0 & 0 & 0 & 0 & -1 & 2+p^{2}
\end{array}\right)^{-1}
$$

Subsequently, by computing the matrix inverse the following pivotal elements are defined

$$
\begin{gathered}
\hat{G}_{11}^{(1)}=\frac{\left(p^{4}+4 p^{2}+2\right)\left(p^{4}+6 p^{2}+7\right)}{\left(p^{4}+5 p^{2}+2\right)\left(p^{2}+4\right)\left(p^{2}+3\right)\left(p^{2}+1\right)}, \\
\hat{G}_{119}^{(1)}=\frac{2}{p^{10}+13 p^{8}+61 p^{6}+123 p^{4}+98 p^{2}+24}, \\
\hat{G}_{137}^{(1)}=\frac{1}{p^{6}+8 p^{4}+17 p^{2}+6} .
\end{gathered}
$$

These expressions correspond to $\hat{x}_{\gamma}, \hat{y}_{\gamma}$ and $\hat{z}_{\gamma}$, respectively, in Eqs. (3.11)- (3.17).

The difference between the Sierpinski gasket and Sierpinski carpet devices is that for the Sierpinski gasket inspired transducer the Green function elements for any generation level are obtained through the application of Eq. (2.8). Thus prohibiting the need to calculate the Green function matrices at each generation level and so high generation level results can easily be obtained. Since Eq. (2.8) does not provide accurate results for the Sierpinski carpet devices, the need for the Green function matrices were required. Consequently, only low generation level results are achieved.

\section{APPENDIX B}

\section{Development of Green Function Equations}

Model $\delta$ is concerned with the inclusion of three boundary conditions; one input vertex and two output vertices. For this model the application of Eq. (2.9) resulted in the 
following four equations

$$
\begin{gathered}
x_{\delta}=\hat{x}_{\delta}+\hat{x}_{\delta} b_{1, \delta} x_{\delta}+2 \hat{y}_{\delta} b_{2, \delta} y_{\delta}, \\
y_{\delta}=\hat{y}_{\delta}+\hat{y}_{\delta} b_{1, \delta} x_{\delta}+\hat{w}_{\delta} b_{2, \delta} y_{\delta}+\hat{t}_{\delta} b_{2, \delta} y_{\delta}, \\
t_{\delta}=\hat{t}_{\delta}+\hat{y}_{\delta} b_{1, \delta} y_{\delta}+\hat{w}_{\delta} b_{2, \delta} t_{\delta}+\hat{t}_{\delta} b_{2, \delta} w_{\delta}, \\
w_{\delta}=\hat{w}_{\delta}+\hat{y}_{\delta} b_{1, \delta} y_{\delta}+\hat{w}_{\delta} b_{2, \delta} w_{\delta}+\hat{t}_{\delta} b_{2, \delta} t_{\delta} .
\end{gathered}
$$

Solving these simultaneously resulted in Eqs. (3.7) - (3.10).

For model $\gamma$ Eqs. (3.11) - (3.17) are found by applying Eq. (2.9) and accounting for the lattice symmetries when boundary conditions are neglected;

$$
\begin{gathered}
\hat{G}_{11}^{(n)}=\hat{G}_{1919}^{(n)}=\hat{G}_{3737}^{(n)}=\hat{G}_{5555}^{(n)}, \\
\hat{G}_{119}^{(n)}=\hat{G}_{155}^{(n)}=\hat{G}_{1937}^{(n)}=\hat{G}_{3755}^{(n)}, \\
\hat{G}_{137}^{(n)}=\hat{G}_{1955}^{(n)} .
\end{gathered}
$$

However these symmetries do not hold when boundary conditions are accounted for. These Green function elements are associated with the internal structure of the lattice and as a result it is possible to set these elements equal to one another, as shown in Eqs. (B5) - (B7). The boundary conditions take into account the backing layer and mechanical loads set at the input/ output vertices. This then results in

$$
\begin{gathered}
G_{119}^{(n)}=G_{155}^{(n)}, \\
G_{1937}^{(n)}=G_{3755}^{(n)}, \\
G_{11}^{(n)} \neq G_{1919}^{(n)} \neq G_{3737}^{(n)}, \\
G_{119}^{(n)} \neq G_{137}^{(n)} .
\end{gathered}
$$


The use of Eq. (2.9) results in seven equations,

$$
\begin{gathered}
x_{\gamma}=\hat{x}_{\gamma}+\hat{x}_{\gamma} b_{1, \gamma} x_{\gamma}+2 \hat{y}_{\gamma} b_{2, \gamma} y_{\gamma}+\hat{z}_{\gamma} b_{2, \gamma} z_{\gamma}, \\
y_{\gamma}=\hat{y}_{\gamma}+\hat{y}_{\gamma} b_{1, \gamma} x_{\gamma}+\hat{x}_{\gamma} b_{2, \gamma} y_{\gamma}+\hat{y}_{\gamma} b_{2, \gamma} z_{\gamma}+\hat{z}_{\gamma} b_{2, \gamma} y_{\gamma}, \\
z_{\gamma}=\hat{z}_{\gamma}+\hat{z}_{\gamma} b_{1, \gamma} x_{\gamma}+2 \hat{y}_{\gamma} b_{2, \gamma} y_{\gamma}+\hat{x}_{\gamma} b_{2, \gamma} z_{\gamma}, \\
u_{\gamma}=\hat{y}_{\gamma}+\hat{z}_{\gamma} b_{1, \gamma} y_{\gamma}+\hat{y}_{\gamma} b_{2, \gamma} w_{\gamma}+\hat{x}_{\gamma} b_{2, \gamma} u_{\gamma}+\hat{y}_{\gamma} b_{2, \gamma} t_{\gamma}, \\
t_{\gamma}=\hat{z}_{\gamma}+\hat{y}_{\gamma} b_{1, \gamma} y_{\gamma}+\hat{x}_{\gamma} b_{2, \gamma} t_{\gamma}+\hat{y}_{\gamma} b_{2, \gamma} u_{\gamma}+\hat{z}_{\gamma} b_{2, \gamma} w_{\gamma}, \\
w_{\gamma}=\hat{x}_{\gamma}+\hat{y}_{\gamma} b_{1, \gamma} y_{\gamma}+\hat{x}_{\gamma} b_{2, \gamma} w_{\gamma}+\hat{y}_{\gamma} b_{2, \gamma} u_{\gamma}+\hat{z}_{\gamma} b_{2, \gamma} t_{\gamma}, \\
v_{\gamma}=\hat{x}_{\gamma}+\hat{z}_{\gamma} b_{1, \gamma} z_{\gamma}+2 \hat{y}_{\gamma} b_{2, \gamma} u_{\gamma}+\hat{x}_{\gamma} b_{2, \gamma} v_{\gamma}
\end{gathered}
$$

which again are solved simultaneously to produce Eqs. (3.11) - (3.17). 